\title{
Demand-Insensitive Price Relationships in Competitive Private Commons
}

\author{
Emir Kavurmacioglu \\ Division of Systems \\ Engineering \\ Boston University \\ Boston, MA 02135 \\ emir@bu.edu
}

\author{
Murat Alanyali \\ Department of Electrical \\ Boston University \\ Boston, MA 02135 \\ alanyali@bu.edu
}

\author{
David Starobinski \\ Division of Systems \\ Engineering \\ Boston University \\ Boston, MA 02135 \\ staro@bu.edu
}

\begin{abstract}
We introduce an economic model for private commons that consists of network providers serving a fixed primary demand and making strategic pricing decisions to improve their revenues by providing service to a secondary demand. For general forms of secondary demand, we establish the existence and uniqueness of two critical prices for each provider: the break-even price and the market sharing price. The prior determines service profitability while the latter determines a provider's willingness to share the market. We further show that the market sharing price is always greater than the break-even price, leading to a price interval in which a provider is both profitable and willing to share the market. Making use of these results, we shed insight into the nature of market outcomes (Nash equilibria) when two providers compete to attract secondary demand: (i) if the market sharing intervals of the two providers overlap, then the providers end up sharing the market; (ii) else, the provider with the lower break-even price captures the entire market as the result of a price war.
\end{abstract}

\section{INTRODUCTION}

In an effort to make more efficient usage of the electromagnetic spectrum, the FCC is promoting new paradigms for spectrum sharing. One such paradigm is the Private Commons, which is deemed both "commercially viable and technologically feasible" [1]. This paradigm supports spectrum transactions, where ownership of spectrum remains with the license holder providing service to its primary users, but this provider may also provide spectrum access to secondary users for a fee. The Amazon's Kindle model can be viewed as an early realization of this paradigm, in which owners of Kindle e-readers make secondary use of AT\&T network to retrieve contents from the cloud. Other precursors include machine to machine (M2M) communication and mobile virtual network operators (MVNOs), such as Republic Wireless, that mainly rely on $\mathrm{Wi}-\mathrm{Fi}$ and utilize a cellular network as a fallback.

A network providing secondary spectrum access has two major challenges to resolve. The first such challenge

Copyright is held by author/owner(s). is to keep a profitable margin by making the correct strategic pricing decision. The difficulty of this challenge lies with the uncertainty in the demand response to the advertised price, which is generally stipulated by a so-called demand function. However, this function is hard to characterize and may also be time-varying.

The second challenge is the market competition that a provider faces in spectrum offerings. When several providers offer secondary spectrum provisions, this opens up the possibility of market sharing by advertising the same price. However, it is not clear whether such an action is favorable over trying to capture the entire market by slightly lowering the price in turn.

The main goal of our paper is to provide insight, for general demand functions, into the market outcomes of a game involving multiple providers offering secondary spectrum access in their private commons. Toward this end, we identify two price thresholds playing a critical role for each provider, and establish a fundamental relationship between them.

We first prove the existence of a unique break-even price $p^{B E}$ that guarantees a positive profit as long as a provider sets its price above it. Next, we derive another unique threshold price, called market sharing price $p^{M S}$, below which a provider finds it desirable to share secondary demand with another provider. Even though the expressions for the market sharing and break-even prices are implicit, we prove that the market sharing price is always strictly greater than the break-even price, regardless of the demand function. This leads to the conclusion that there always exists a price interval in which a network provider is profitable and is willing to share the market as illustrated in Fig. 1(a).

Finally, we consider a duopoly competition where network providers make pricing decisions to maximize their revenues. We formally establish the best response strategy of each provider and list the possible market outcomes in the form of Nash equilibria. Due to space limitations, the proofs of all the lemmas and theorems presented in this paper can be found in [2].

\section{MODEL}

We consider two spectrum providers, where each provider $i$ has a capacity $C_{i}$ and a primary demand of volume $\lambda_{i}$, which generates a revenue of $K_{i}$ units per service. These providers compete for a stream of secondary demand, whose volume depends of their pricing 


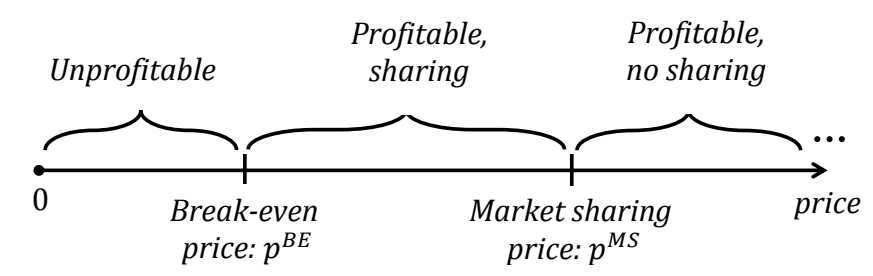

(a) Illustration of profitable and market sharing price intervals

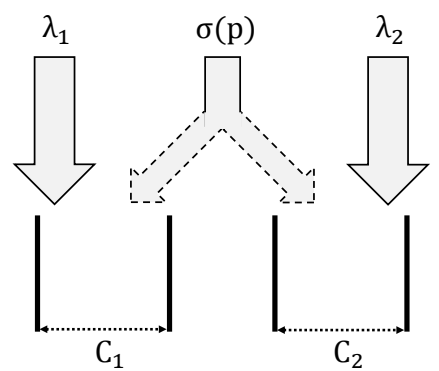

(b) Market model of two providers

Figure 1: Price intervals and traffic flow model

of secondary service as illustrated in Figure 1(b). We assume a traffic model where if provider $i$ receives a total demand of volume $\lambda_{i}$, then it can accommodate the volume $\min \left(C_{i}, \lambda_{i}\right)$. The excess demand $\max \left(\lambda_{i}-C_{i}, 0\right)$ does not generate any revenue for the provider.

The total demand of a provider consists of its primary demand and, depending on its pricing and the pricing of its competitor, a secondary demand. The steadystate primary and secondary demands, $\lambda_{i}$ and $\sigma(p)$, and the overflow assumption are consistent with fluid models Such models have widely been used in the literature to characterize network traffic at the flow level [3].

We shall assume that the two demand types access the capacity in an uncoordinated fashion, as suggested by documentation on private commons [1]. Specifically, the two types of demand share capacity on equal basis, such that if the demand of provider $i$ is composed of two types with respective volumes $\lambda_{i}$ and $\sigma_{i}$, then the overflow volume of each type is proportional to the intensity of demand of that type. That is, in view of our previous assumption, a fraction $\min \left(1, \frac{C_{i}}{\lambda_{i}+\sigma_{i}}\right)$ of each type of demand is actually accommodated by the provider.

We denote the price that provider $i$ charges per unit of serviced secondary demand by $p_{i}$. The volume of the secondary demand is assumed to be determined by the minimum price $\min \left(p_{1}, p_{2}\right)$ stipulated by the two providers. Specifically, the volume of secondary demand is $\sigma\left(\min \left(p_{1}, p_{2}\right)\right)$, where $\sigma(\cdot)$ is the demand function. We make the mild assumption that this function is differentiable and decreasing $\left(\frac{\partial}{\partial p} \sigma(p) \leq 0\right)$. We shall also assume that $\sigma(0)>0$ and $\lim _{p \rightarrow \infty} \sigma(p)=0$.

It is assumed that the secondary demand is attracted to the provider that charges the lowest price. This behavior can be explained by price aversion, a concept employed in marketing management [5]. When both providers charge the same price, the resulting secondary demand splits between the two providers according to an arbitrary probability vector $\left[\alpha_{1}, \alpha_{2}\right]$ such that $\alpha_{1}+\alpha_{2}=1$ and $\alpha_{1}, \alpha_{2}>0$. Namely, each provider $i$ receives a secondary demand of volume $\alpha_{i} \sigma\left(p_{i}\right)$.

Hence, if provider $i$ receives a secondary demand of volume $\sigma\left(p_{i}\right)$, its overall revenue is given by:

$$
\begin{aligned}
W_{i}\left(p_{i}, \sigma\left(p_{i}\right)\right) \triangleq p_{i} \sigma\left(p_{i}\right) \min \left(1, \frac{C_{i}}{\lambda_{i}+\sigma\left(p_{i}\right)}\right) \\
+K_{i} \lambda_{i} \min \left(1, \frac{C_{i}}{\lambda_{i}+\sigma\left(p_{i}\right)}\right) .
\end{aligned}
$$

In this case, the secondary profit rate of the provider is:

$$
\Pi_{i}\left(p_{i}, \sigma\left(p_{i}\right)\right) \triangleq W_{i}\left(p_{i}, \sigma\left(p_{i}\right)\right)-W_{i}(0,0) .
$$

Since the secondary demand that a provider receives depends on the prices of both providers, so does the profit of the provider. We define the reward $R_{i}\left(p_{i}, p_{-i}\right)$ of provider $i$ as its profit when it charges secondary access $p_{i}$ and its competitor charges $p_{-i}$ units. Namely,

$$
R_{i}\left(p_{i}, p_{-i}\right) \triangleq \begin{cases}\Pi_{i}\left(p_{i}, \sigma\left(p_{i}\right)\right) & \text { if } p_{i}<p_{-i} \\ \Pi_{i}\left(p_{i}, \alpha_{i} \sigma\left(p_{i}\right)\right) & \text { if } p_{i}=p_{-i} \\ \Pi_{i}\left(p_{i}, 0\right) & \text { if } p_{i}>p_{-i}\end{cases}
$$

In the interest of space, the discussion of this paper is limited to the case when each provider's network is underloaded prior to inclusion of any secondary demand, that is $\lambda_{i}<C_{i}$, but can be overloaded for low enough prices, that is $\lambda_{i}+\sigma(0)>C_{i}$.

\section{MAIN RESULTS}

In this section we present our main results on demandinvariant price relationships in a secondary spectrum markets. Since the results apply to each provider separately, we drop the index $i$ for notational simplicity.

We define the break-even price $p^{B E}(\alpha)$ as the price at which the profit of a provider is zero when it attracts a fraction $0<\alpha \leq 1$ of the total demand, namely $\Pi\left(p^{B E}, \alpha \sigma\left(p^{B E}\right)\right)=0$. We require $\sigma\left(p^{B E}\right)>0$ to rule out prices that do not generate any secondary demand.

We next define the market sharing price $p^{M S}$, that asserts whether a provider finds it desirable to share the secondary demand or not. Specifically, let

$$
\Delta \Pi(p) \triangleq \Pi(p, \alpha \sigma(p))-\Pi(p, \sigma(p)) .
$$

Then, $\Delta \Pi\left(p^{M S}\right)=0$. Analogous to the relationship between the break-even price and provider profitability, a provider finds it undesirable to share the secondary demand at prices above the market sharing price, whereas the opposite is true for prices below the market sharing 
price. Having defined the break-even and market sharing prices, we can proceed with stating our main results in the following theorem:

Theorem 3.1 (Market Sharing Interval) For any secondary demand function, satisfying the assumptions described in Section 2 and for all values of $\alpha: 0<\alpha \leq$ 1 , there exists a price interval

$$
(\mathcal{P}) \equiv\left(p^{B E}(\alpha), p^{M S}\right),
$$

such that for all $p \in(\mathcal{P}): \Pi(p, \alpha \sigma(p))>\Pi(p, \sigma(p))>0$.

Theorem 3.1 states that no matter the specific shape of a secondary demand function, the existence of the price interval $(\mathcal{P})$ at which a network provider is profitable and finds it preferable to share the secondary demand is guaranteed. In the next two subsections, we outline how the inequalities stated in Theorem 3.1 are obtained.

\subsection{Profitability and Break-Even Price}

In this section we seek to analyze a provider's profit and the resulting break-even price. Our result applies both to the cases when a network provider serves the entire secondary demand (i.e., $\alpha=1$ ) and when it shares the market with another provider (i.e, $\alpha<1$ ).

The following theorem, provides an equation that allows the computation of the break-even price $p^{B E}(\alpha)$ for the aforementioned values of $\alpha$. The theorem also establishes the uniqueness of this price and the region of profitable prices.

\section{Theorem 3.2 (Break-Even Price)}

1. For a given $0<\alpha \leq 1$, such that $\lambda+\alpha \sigma(0)>C$ :

(a) A break-even price $p^{B E}(\alpha)$ is a solution to the following equation ${ }^{1}$ :

$$
p=\frac{(\alpha \sigma(p)+\lambda-C) \lambda K}{C \alpha \sigma(p)} .
$$

(b) The break-even price $p^{B E}(\alpha)$ is unique.

(c) The profit of a provider is such that:

$$
\begin{array}{ll}
\Pi(p, \sigma(p))>0 & \text { if } p>p^{B E}(\alpha) \\
\Pi(p, \sigma(p))<0 & \text { if } p<p^{B E}(\alpha) .
\end{array}
$$

2. For a given $0<\alpha<1$, such that $\lambda+\alpha \sigma(0) \leq C$, the break-even price $p^{B E}(\alpha)$ is 0 .

Thus, we have effectively formulated and characterized the unique break-even price that determines a network provider's profitability.

\subsection{Market Sharing}

In the next theorem, we present our result on how market sharing affects a provider's profit. The theorem establishes the existence and uniqueness of the market sharing price $p^{M S}$ and provides an implicit equation to compute it.

\footnotetext{
${ }^{1}$ This implicit equation can be solved with wellestablished fixed point iterations, such as Newton's Method.
}

Theorem 3.3 (Market Sharing Price) For any network provider there exists a unique market sharing price $p^{M S}$, which satisfies the following:

1. If $\lambda+\alpha \sigma(K) \leq C, p^{M S}$ is the solution to:

$$
p=\frac{(\lambda+\sigma(p)-C) \lambda K}{(C-\alpha(\lambda+\sigma(p))) \sigma(p)} .
$$

2. If $\lambda+\alpha \sigma(K)>C$,

$$
p^{M S}=K .
$$

For any given $p^{M S}$, the following is true:

$$
\begin{array}{ll}
\Delta \Pi(p)>0 & \text { for } p<p^{M S} ; \\
\Delta \Pi(p)<0 & \text { for } p>p^{M S} .
\end{array}
$$

Theorem 3.3 yields a rather non-straightforward result such that for any network provider there exists a unique price which acts as a threshold value: market sharing at all prices greater than this threshold results in a profit decrease, while at prices below this threshold the network provider is guaranteed a profit increase by decreasing its secondary demand.

Theorems 3.2 and 3.3 provide implicit equations for the break-even price $p^{B E}(\alpha)$ and market sharing price $p^{M S}$ that depend on the demand function $\sigma(p)$. Strikingly, one can show through careful analysis that the ratio of $p^{B E}(\alpha)$ to $p^{M S}$ is strictly smaller than 1 for any demand function, hence proving Theorem 3.1.

\subsection{Duopoly Competition}

In this section, we consider a duopoly where two provid-ers compete to enhance their profits by first capturing and then serving the secondary demand. To identify a market equilibrium, we utilize the concept of Nash equilibrium from game theory. Since Nash equilibria are classically determined by best response functions, we will first seek to establish the best response dynamics of provider $i$ to a fixed competitor price $p_{-i}$, where the notation $-i$ signifies the competing provider.

Facing a competitor price $p_{-i}$, the strategies available to provider $i$ consist of either matching this price and sharing the secondary demand or not matching it and trying to capture all of the secondary demand. While setting the price below or above the competitor's price follows a rather straightforward approach, the case of matching the competitor's price requires a more detailed analysis due to the discontinuity in the profit function. The next lemma states that if it is possible to increase the profit by capturing all of the secondary demand $\sigma\left(p_{i}\right)$ at a certain price $p_{i}$, then it is also desirable to capture the secondary demand at a slightly lower price $p_{i}^{\prime}<p_{i}$. We will then utilize this result in establishing provider $i$ 's best response for prices $p_{i}>p_{i}^{M S}$.

Lemma 3.1 For any $p_{i}$ such that $\Delta \Pi_{i}\left(p_{i}\right)<0$ holds, there exists a price $p_{i}^{\prime}$ such that $p_{i}^{M S}<p_{i}^{\prime}<p_{i}$ and

$$
\Pi_{i}\left(p_{i}^{\prime}, \sigma\left(p_{i}^{\prime}\right)\right)>\Pi_{i}\left(p_{i}, \alpha_{i} \sigma\left(p_{i}\right)\right) .
$$

The next theorem presents provider $i$ 's best response: 


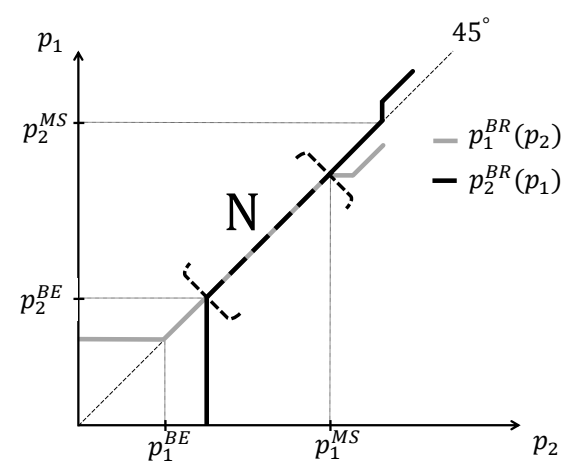

(a) The placement of Nash equilibria on the best response curves when market sharing intervals overlap, corresponding to part 1 of Theorem 3.5, where $p_{1}^{B E}<p_{2}^{B E}$ and $p_{1}^{M S}<p_{2}^{M S}$.

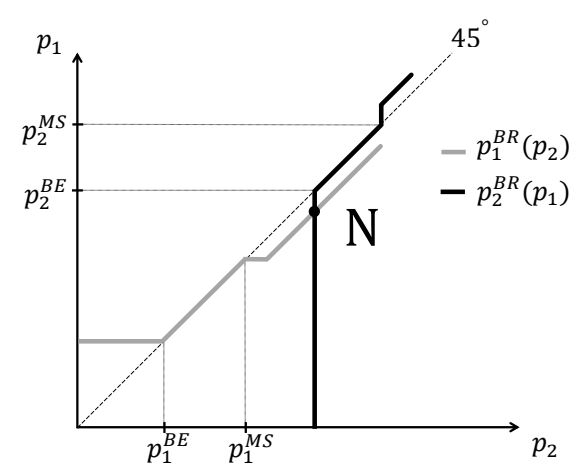

(b) The placement of Nash equilibria on the best response curves when market sharing intervals do not overlap, corresponding to part 2 of Theorem 3.5, where $p_{1}^{M S}<p_{2}^{B E}$.

Figure 2: Illustration of the two possible types of market outcomes.

Theorem 3.4 (Best Response) Provider $i$ best response to its competing provider pricing decision $p_{-i}$ is:

$$
p_{i}^{B R}\left(p_{-i}\right)= \begin{cases}p_{-i}-\epsilon & \text { for } p_{-i}>p_{i}^{M S} \\ p_{-i} & \text { for } p_{i}^{B E}\left(\alpha_{i}\right) \leq p_{-i} \leq p_{i}^{M S} \\ p_{i}^{B E} & \text { for } p_{-i}<p_{i}^{B E}\left(\alpha_{i}\right),\end{cases}
$$

where $\epsilon>0$ is a constant such that $p_{i}^{\prime}=p_{-i}-\epsilon<p_{-i}$ satisfies Eq. (11) in Lemma 3.1 $1^{2}$.

Theorem 3.4 establishes that for any network provider, a price interval, in which market sharing is the best response, is guaranteed to exist. Above this price interval, a provider will lower its price below the competitor's price, as in a typical price war. Below this price interval, profitability conditions from Section 3.1 are violated. While this interval is guaranteed to exist, whether the market equilibrium is established in this interval warrants further analysis. In the next theorem, we determine the different market outcomes by providing the resulting Nash equilibrium from the best response functions of the two providers.

Theorem 3.5 (Nash Equilibrium) In a market with two network providers, a pricing strategy profile $\left(p_{1}^{*}, p_{2}^{*}\right)$ is a Nash equilibrium such that:

1. If $\max \left(p_{1}^{B E}\left(\alpha_{1}\right), p_{2}^{B E}\left(\alpha_{2}\right)\right) \leq \min \left(p_{1}^{M S}, p_{2}^{M S}\right)$, then $p_{1}^{*}=p_{2}^{*}$, and for $i=1,2$

$$
p_{i}^{*} \in\left[\max \left(p_{1}^{B E}\left(\alpha_{1}\right), p_{2}^{B E}\left(\alpha_{2}\right)\right), \min \left(p_{1}^{M S}, p_{2}^{M S}\right)\right] .
$$

2. If $\max \left(p_{1}^{B E\left(\alpha_{1}\right)}, p_{2}^{B E}\left(\alpha_{2}\right)\right)>\min \left(p_{1}^{M S}, p_{2}^{M S}\right)$ and without loss of generality $p_{1}^{B E}\left(\alpha_{1}\right)<p_{2}^{B E}\left(\alpha_{2}\right)$

$$
p_{1}^{*}=p_{2}^{B E}\left(\alpha_{2}\right)-\epsilon \text { and } p_{2}^{*}=p_{2}^{B E}\left(\alpha_{2}\right),
$$

where $\epsilon$ is defined as in Theorem 3.4.

Interpretation of the Nash equilibria. As stated in Theorem 3.5, the exact price profiles that give the Nash equilibria depend on the relationship between the market sharing intervals of the two providers. If two price

\footnotetext{
${ }^{2}$ For continuous prices, a well-known approach used in game theory is to assume that each provider's price is a multiple of a small discretization step $\epsilon$ [4].
}

intervals overlap, as illustrated in part (a) of Fig. 2, any equal price pair in that interval will give us a Nash equilibria. As a result, two providers share the market and set their prices at a value above their respective breakeven prices but always less than the smaller of the two market sharing prices, a value which is guaranteed to be no greater than $K_{i}$, the primary reward collected.

On the other hand, if the market sharing price intervals of the two providers do not intersect, as illustrated in part (b) of Fig. 2, the market outcome is the same as the result of a price war, where the provider with the lower break-even price captures all of the secondary demand by pricing slightly below its competitor's breakeven price. The losing provider cannot match this price without making a negative profit. In this case, even though both providers find it desirable to go into market sharing as the prices approach their break-even prices, the gap between the two market sharing intervals does not allow them to converge to a market sharing point.

Acknowledgements: This work was supported, in part, by the US National Science Foundation grant CCF0964652.

\section{REFERENCES}

[1] M.M. Buddhikot. Understanding dynamic spectrum access: Models,taxonomy and challenges. In DySPAN, pages 649 -663, 2007.

[2] E. Kavurmacioglu, M. Alanyali, and D. Starobinski. Provider competition and price equilibria in private commons. CISE Tech. Report 2012-IR-0007, Boston University, July 2012.

[3] F.P. Kelly and R.J. Williams. Fluid model for a network operating under a fair bandwidth-sharing policy. Annals of Applied Probability, pages 1055-1083, 2004.

[4] M. J. Osbourne. An Introduction to Game Theory. Oxford University Press, New York, NY, USA, 1st edition, 2004.

[5] G. J. Tellis and G. J. Gaeth. Best value, price-seeking, and price aversion: The impact of information and learning on consumer choices. Journal of Marketing, 54(2):pp. 34-45, 1990. 